\title{
Lipids in the Piezo1 channel pore: gating mechanism, or simulation artifact?
}

\author{
Wenjuan Jiang ${ }^{1}$, Jerome Lacroix ${ }^{2 *}$, Yun Lyna Luo ${ }^{1 *}$ \\ ${ }^{1}$ College of Pharmacy, Western University of Health Sciences, Pomona, CA 91709 \\ ${ }^{2}$ Graduate College of Biomedical Sciences, Western University of Health Sciences, Pomona, CA 91709
}

Corresponding emails:

jlacroix@westernu.edu; luoy@westernu.edu 


\section{ABSTRACT}

Opening and closure of certain mechanosensitive ion channels has recently been linked with the presence of lipids in or near their pores. Although non-conducting structures of mechanosensitive Piezo channels do not show the presence of lipids in the pore, computational simulations suggest whole phospholipids enter the Piezo1 pore in the closed state. Here, to probe this phenomenon, we conduct coarse-grained (CG) and all-atom (AA) simulations of Piezo1 with different solvation algorithms and equilibrium protocols, including CG-to-AA reverse mapping from Martini CG force field to CHARMM AA force field. Our results show that the lack of initial hydration of the upper pore region, enabled by common CG but not AA solvation algorithms, allows entry of whole lipids through gaps between pore helices during subsequent equilibrium simulations. Absolute binding free energy calculations show that these lipids are thermodynamically unfavorable, indicating they are likely kinetically trapped in the pore during microsecond-long AA simulations. An alternative equilibrium protocol is proposed to avoid such simulation artifact for channels whose pores are walled with transmembrane gaps. This work underscores the notion that, as simulated systems become increasingly complex, interpretation of simulated data in physiological contexts requires extra precautions. When no experimental data is available, free energy approaches such as those implemented here appear as trustworthy validations of results observed from MD trajectories.

\section{SIGNIFICANCE STATEMENT}

Membrane-embedded proteins constantly interact with lipid molecules. Computational molecular dynamics simulations have become an indispensable tool for investigating the role of such protein-lipid interactions. Recent simulation studies suggest the presence of lipids in the nonconducting pore of mechanosensitive Piezo1 channels. Here, we show that certain computational protocols at the initial equilibrium stage enable lipids to be trapped in the pore despite a large free energy cost, suggesting these lipids are kinetically trapped and likely entered the pore as a result of a computational artifact rather than from a physiological process. This work emphases the need for additional validations of membrane protein simulation outcomes and proposes alternative protocols to avoid such artifact. 


\section{INTRODUCTION}

Computational molecular dynamics (MD) simulation has become a powerful and indispensable tool to study ion channels and understand molecular mechanisms underlying conduction, selectivity, and gating. Thanks to advances in X-ray crystallography, cryogenic electron microscopy (cryo-EM), and artificial intelligence-driven structure prediction algorithms such as AlphaFold v2.0 and RoseTTAFold, the number of membrane protein structures has largely increased over the past few years. A comparable increase in MD simulations is thus needed to study how membrane protein structures, obtained in extreme physical conditions, equilibrate in the physiological environment of hydrated membranes at body temperature, as well as to identify and validate functional states captured by these structures, to derive new functional state models, and/or to map conformational transitions between states by free energy calculations.

Various MD simulation engines are publicly available, and their performance have been constantly improved on GPU cards or massively paralleled supercomputers. Each simulation engine also comes with its own system preparation tools for solvation, membrane embedding, and a set of standard equilibrium protocols. In recent year, multiscale MD simulations using both coarse-grained (CG) and all-atom (AA) have become popular in membrane protein simulations. This is because Martini CG models significantly accelerate lipid diffusion $(1,2)$ and thus enable membrane topology change and lipid binding/unbinding an order of magnitude faster than in AA simulations. However, CG models require using protein tertiary structure constraints, which is far from ideal for investigating protein conformational change. Therefore, back-mapping membrane protein models obtained from converged CG simulations to all-atom models (i.e., reverse-mapping) provides a clever and efficient solution to model how lipids influence protein dynamics at atomistic resolution(3).

This hybrid CG-equilibrium-AA-production protocol is especially useful for mechanosensitive Piezo channels, which represent some of the largest plasma membrane proteins known to date $(4,5)$. When embedded in lipid bilayer, Piezo channels are anticipated to curve the surrounding membrane into an inverted-dome shape(6). The first all-atom simulations of Piezo1 indicate that the resting conformation of Piezo1 indeed curves the surrounding bilayer and the curvature stabilizes over $3 \mu \mathrm{s}(7)$. While in a CG simulation, this was achieved within hundreds of nanoseconds with significantly reduced computational resources(8). Force-induced Piezo1 opening has been computationally observed by two independent groups, including ours $(8,9)$. In both studies, CG-MD was first conducted until membrane curvature reached equilibrium with fixed protein backbone. The last snapshot of the CG simulation was then reverse-mapped back to AA model for simulating Piezo1 conformational change either through reducing 
membrane curvature(8) or increasing membrane tension(9). Both approaches consistently revealed widening of the Piezo1 pore upon Piezo1 arm flattening. The open conformation from the former study was further validated against experimental ionic conductance, selectivity, and mutant phenotypes(8).

One common observation from both studies was the presence of multiple lipids inside the nonconducting Piezo1 pore during CG simulations. After reverse-mapping CG to AA model, those lipids either remained in the pore during three replicas of $\sim 2 \mu$ s $A A$ simulations(8), or diffuse away from the pore under substantially large membrane tension of 40 bar(9). Notably, pore occlusion by lipids has emerged to be a novel paradigm of gating mechanosensitive channels such as the $E$. coli smallconductance mechanosensitive channel $\operatorname{MscS}(10)$ and the human TWIK-related arachidonic acidactivated potassium (TRAAK) channel(11). However, no electron density was observed in the nonconducting pore of Piezo1 structures. In addition, our first AA simulation of Piezo1 directly prepared from AA model did not show permeant lipid occlusion of the pore during $8 \mu$ s simulations(7). Thus, inconsistencies regarding the presence of lipids in the pore from MD simulations could originate from subtle differences in CG and AA force fields (such as the balance among lipid-water-protein interactions), or from differences in system preparation and equilibrium protocols. Because the presence of lipids in the channel pore would radically change our understanding of Piezo channels, it is critical to determine how lipids enter the Piezo1 pore during MD simulations and whether these lipids are thermodynamically favorable inside the pore.

To answer these questions, we tested multiple Piezo1 simulation protocols. Water and lipid densities in the Piezo1 pore obtained from CG simulations were compared with those obtained from AA equilibrated conformations and from CG-to-AA reverse-mapped conformations. We found that the presence of lipids inside the Piezo1 pore strongly depends on the solvation algorithms. While the solvation algorithm from all-atom simulation allows water molecules to fill in the available space in the pore(12), the algorithm in INSANE.py, commonly used to prepare CG models, only adds water molecules above and below the lipid bilayer, leaving a vacuum inside the pore(13). Consequently, during the CG equilibrium stage, the pore becomes rapidly populated by lipids or water, depending on whether a restrain is applied to the lipid headgroups or to the whole lipids, respectively. This discrepancy introduced during CG equilibrium persists throughout the subsequent $1 \mu \mathrm{s}$ A production runs and is reproducible in two independent replicas. To provide a quantitative conclusion, we computed the absolute binding free energy of the lipids in the Piezo1 pore vs. in the bulk bilayer and confirmed that the presence of lipids in the pore conformation solved in cryo-EM structure is extremely unfavorable $(\Delta G=+45.9 \pm 0.9 \mathrm{kcal} / \mathrm{mol})$. 
Therefore, we conclude that the lipid headgroups positioned inside the nonconducting Piezo1 pore during CG equilibrium are thermodynamically unfavorable, and thus are kinetically trapped during microseconds of $A A$ simulation.

This study highlights the sensitivity of MD simulation outcomes to the initial condition used to prepare and equilibrate membrane protein systems. As the simulated system becomes more and more complex, interpretation of simulated data requires careful consideration of experimental evidence. Critically, when experimental data is unavailable, free energy calculations constitutes a robust thermodynamic validation of the results obtained from MD simulations.

\section{RESULTS}

\section{Water and lipid distribution along the Piezo1 pore region}

To understand how lipids enters the Piezo1 pore in the nonconducting conformation solved by cryo-EM structure, we constructed a pore model of Piezo1 channel consisting the cap, pore, and CTD domains (residue V1976 to R2546, Figure1a)(6). Piezo1 channel is a homotrimer. At the nonconducting state, the upper and lower pore region is separated by a hydrophobic constriction site consisting of three V2476. Previous simulations have shown that the widening of the construction site is required for the Piezo1 pore to conduct $\mathrm{K}^{+}$ions(8). To quantify the number of lipid headgroups, tails, and water molecules inside the pore during each simulation, we define "upper pore region", "hydrophobic constriction site" and "lower pore region" by the positions of P2455, L2469, V2476 and F2485 on the inner pore helices (TM38), including the linker between the cap and TM38 (Figure 1b). The numbers of CG beads for water, lipid headgroups (called 'PO4' CG bead type) and lipid tails classified into these regions over time (see Methods) are plotted in Figure 2. 
a)

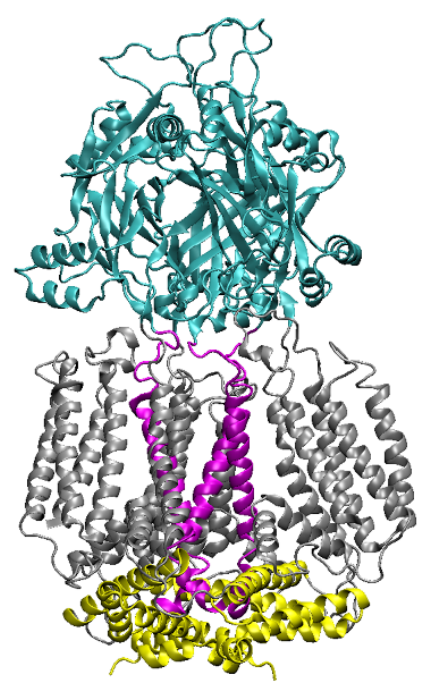

b)

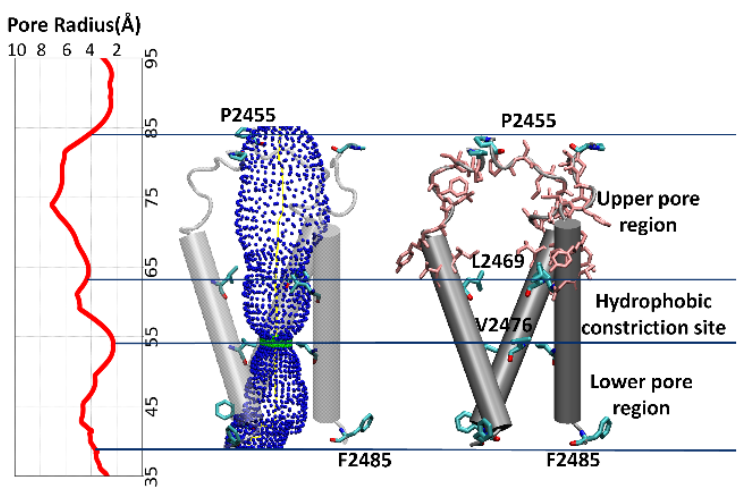

Figure 1. Piezo1 pore model. a) The Piezo1 pore model (residue V1976 to R2546) is mainly composed of the cap colored in cyan, pore in purple, CTD region in yellow, and repeat A in silver. b) Pore radius profile for the cryo-EM solved Piezo1 pore and classified regions defined to count the number of water and lipid molecules inside. The residues P2455, L2469, V2476 and F2485 are in licorice mode. Pore shown in 3D is calculated using HOLE program(14) and plotted in $\operatorname{VMD}(15)$. The pink residues in licorice mode are the hydrophobic residues along the Piezo1 upper pore region.

Two CG equilibrium protocols and two replicas each were conducted. To make the comparison more direct, protein backbone is constrained to the original cryo-EM conformation throughout the equilibrium and production runs using positional restraint (Figure $\mathbf{2}$ and Table S1). In CG model solvation step, solvent molecules were generated using a 3D grid based on a preset solvent radius where the grid cells occupied by membrane and/or proteins were flagged unavailable, and the remaining cells were filled with solvents(13). The default result is that water molecules are added above and below the bilayer, thus left the pore region empty initially (Figure 2a). This solvation step is followed by a standard equilibrium protocol obtained from CHARMM-GUI webserver (Methods and Table S1a), in which water molecules first undergo equilibrium with protein beads and lipid headgroup beads restrained. During this step, we observed that lipid tails enter the upper and lower pore region through inter-subunit opening between three inner pore helices (Figure 3). With the headgroup force constant reduced from $5000 \mathrm{~kJ} / \mathrm{mol} / \mathrm{nm}^{2}$ (step6.1) to $1000 \mathrm{~kJ} / \mathrm{mol} / \mathrm{nm}^{2}$ (step6.5), the CG lipid headgroups gradually moved from outside the inner pore helix to the upper pore region. In the following production run, lipid headgroup restraints are removed, and three lipid headgroups fully entered the upper pore region and remained for the whole $1 \mu \mathrm{s}$ 
bioRxiv preprint doi: https://doi.org/10.1101/2021.11.28.470286; this version posted November 29, 2021. The copyright holder for this preprint (which was not certified by peer review) is the author/funder, who has granted bioRxiv a license to display the preprint in perpetuity. It is made available under aCC-BY-ND 4.0 International license.

production run. There are 13 to 15 lipid tail beads in the upper pore region during two production runs. As a result, water molecules were mostly excluded from the upper pore region. Two independent replicas show that three PO4 beads remained in the upper pore region during $1 \mu$ s production run (Figure $\mathbf{3 b}$ and Figure 2).

a)

CG Initial Set-Up $t=0$

b)

CG headgroup
$\substack{\text { Restraint } \\ t=1 \mu \mathrm{s}}$

c)

CG whole lipid
Restraint
$t=1 \mu \mathrm{s}$
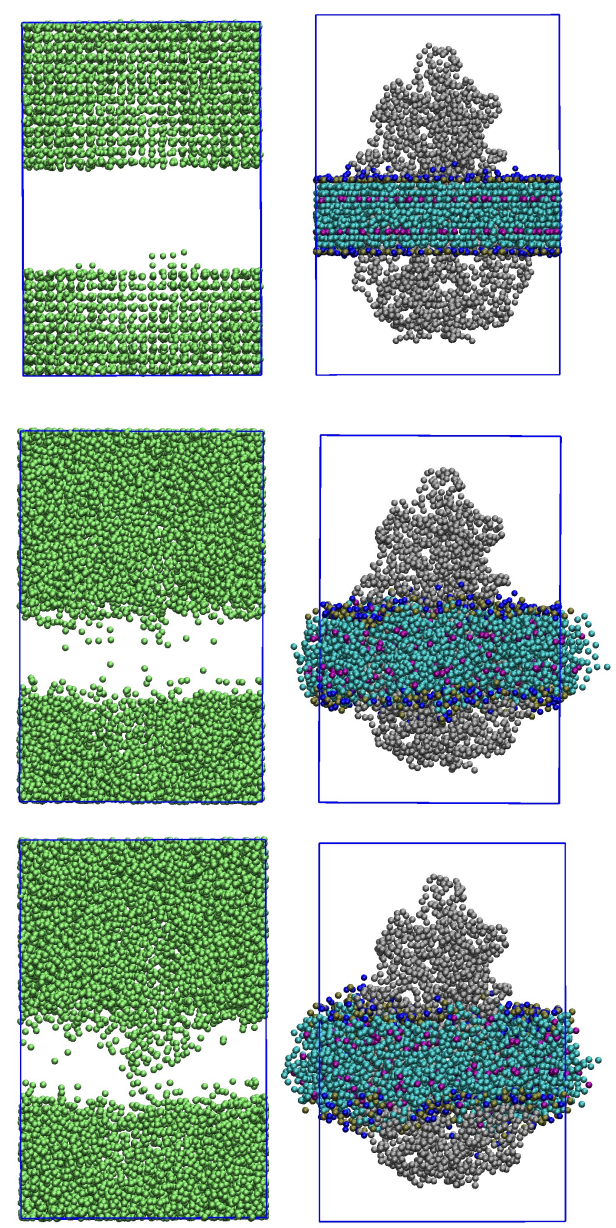
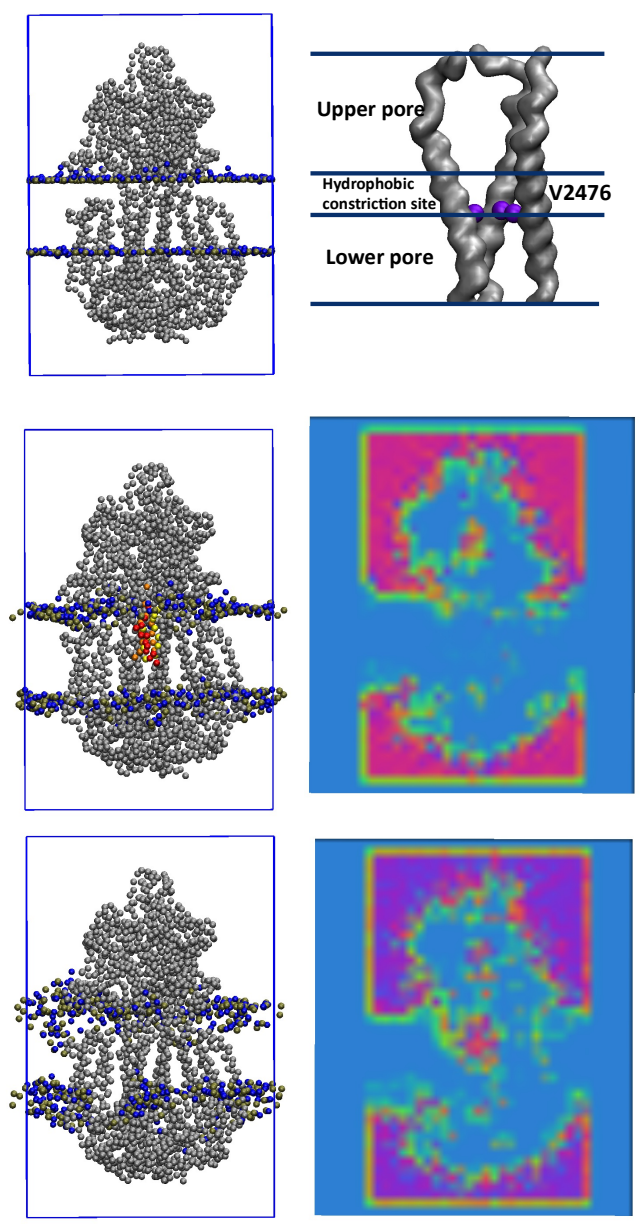

Figure 2. CG simulation of Piezo1 pore domain. a) CG initial system setup by components and a close look of pore region. b) System with lipid headgroup-only restraint during equilibrium stage and fully released bilayer for $1 \mu \mathrm{s}$ production run. c) System with whole lipid restraint during equilibrium stage and fully released bilayer for the $1 \mu \mathrm{s}$ production run. Color scheme: water beads in green, protein backbone in silver, V2476 residue in violet, lipid phosphate group in brown, lipid nitrogen bead in blue, hydrophobic tails in cyan and purple. The 3 lipids trapped during reverse-mapped AA simulations are colored in red, yellow, and orange. All the beads are shown in VDW mode in VMD. The water density map is computed from the last 500 ns for each system and visualized with slice offset $=0.48$ along $x$-axis in VMD. 
The above simulations suggest that the vicinity of the lipids near the empty pore could allow unrestrained lipid tails to enter the pore prior to water molecules. Once the lipid headgroup restraint is removed, those lipid tails quickly pulled their headgroups inside the pore. Following this hypothesis, we restrained the whole lipid during the equilibrium step and found that the water density is greatly increased at the upper pore region. Two replicas show that 20 water beads occupied the upper pore region, and the pore remains fully hydrated during $1 \mu$ s production run (Figure 2 and 3c). During the production run, lipid headgroups were found to enter the pore region sporadically, but do not remain in the pore. This observation is indeed consistent with the previous $8 \mu$ s all-atom simulation prepared directly from AA model instead of from CG to AA model(7). Thus, it appears that the whole lipid restraint setting allowed ample time for water molecules to diffuse into the upper pore region during equilibrium stage, while in the headgroup-only lipid restraint, nearby lipid tail pre-occupied the upper pore region and limited the water accessibility to the entrance throughout the subsequent CG simulations. 


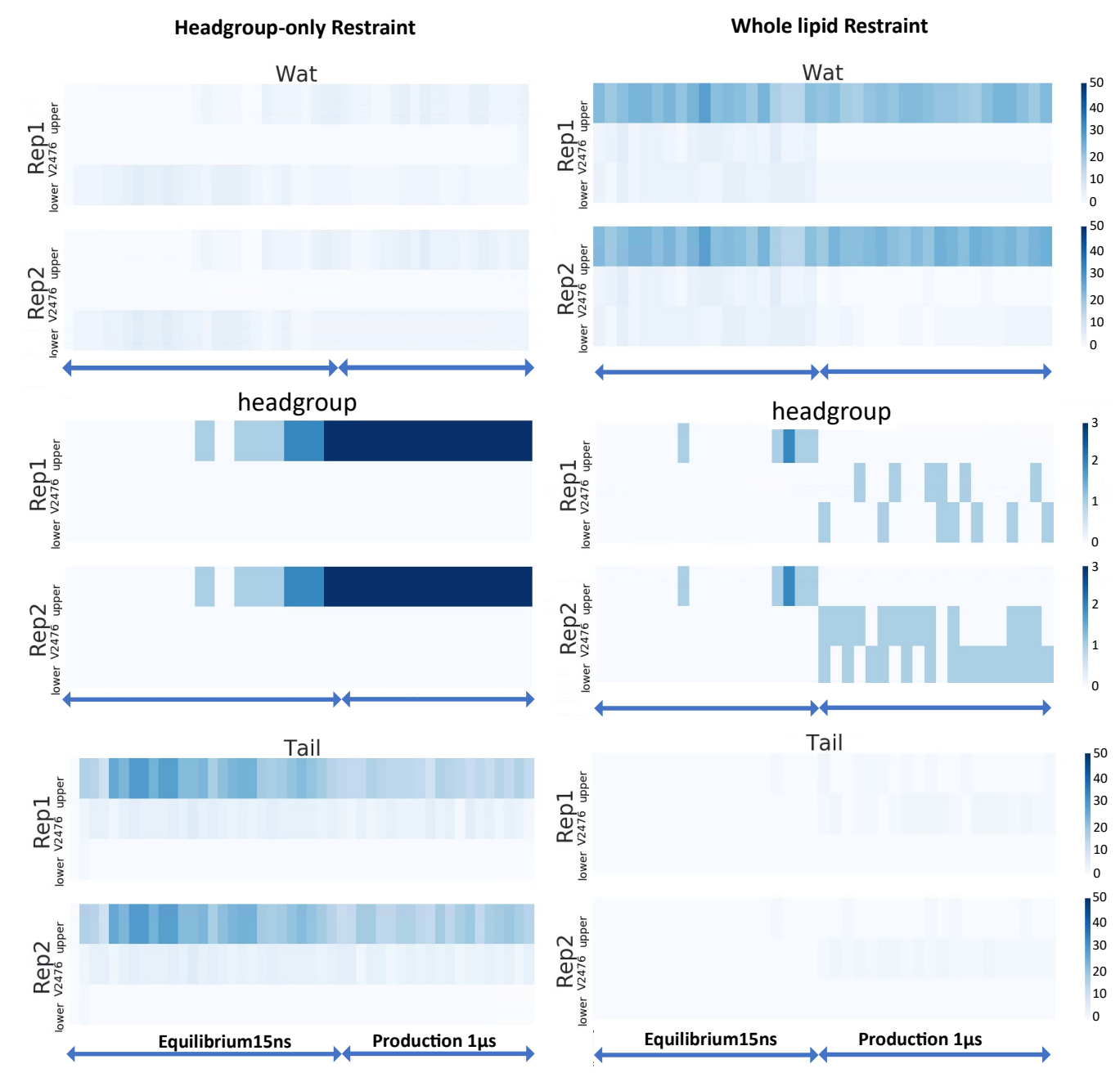

Figure 3. Number of CG water, lipid headgroup, and lipid tail in Piezo1 pore during equilibrium and production runs. In Martini, four water molecules are mapped into one bead, one POPC lipid has 1 headgroup bead PO4 and 8 tail beads. "Headgroup-only restraint" denotes the standard equilibrium protocol with headgroup-only restraint for the bilayer during equilibrium stage, and 'Whole lipid restraint' denotes the equilibrium protocol with the whole lipid atom restraint for the bilayer during equilibrium stage (see Table $\mathbf{S 1}$ for details of the standard equilibrium protocol). The production stage ran for $1 \mu$ s for both CG models with $10000 \mathrm{~kJ} / \mathrm{mol} / \mathrm{nm}^{2}$ position restraints on protein backbone. Each restraint system ran for two replicas of production runs with the same protocol from equilibrium stage, but different initial velocities.

\section{Pore fully hydrated during AA equilibrium with headgroup-only bilayer restraint}

The CG equilibrium tests above suggest that whether the Piezo1 pore is initially solvated, or vacuum dictate the outcome of the following AA simulations. To further validate this point, we prepared an AA system directly without using CG reverse mapping. As the initial AA structure shown in Figure $4 a$, the 
default GROMACS solvation algorithm(16) places water molecules in the empty space of bilayer and protein regions, in contrast to the default INSANE algorithm(13) in CG model which placed no water beads in bilayer and protein regions. Hence, the difference of solvation algorithms between CG and AA models introduced different pore hydration pattern to begin with. Using the standard CHARMM-GUI equilibrium protocol (using headgroup-only restraint at AA equilibrium stage), we found no lipids accumulated at the upper pore region at the end of $1 \mu$ s production run. The AA model water density map shown in Figure $4 \mathbf{b}$ indicated a water density of $0.3 \AA^{-3}$ around $90 \AA<z<100 \AA$ at the upper pore region. The water density decreased to almost zero around $z=76.5 \AA$, which is at the hydrophobic constriction site of the pore, consistent with the nonconducting state of Piezo1 captured by cryo-EM and a previous $8 \mu$ sA simulation(7).

a)

AA Initial Set-Up $\mathbf{t}=\mathbf{0}$

b)

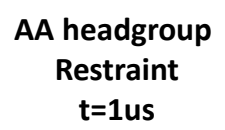

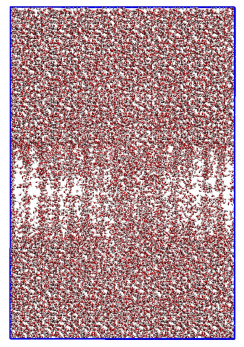
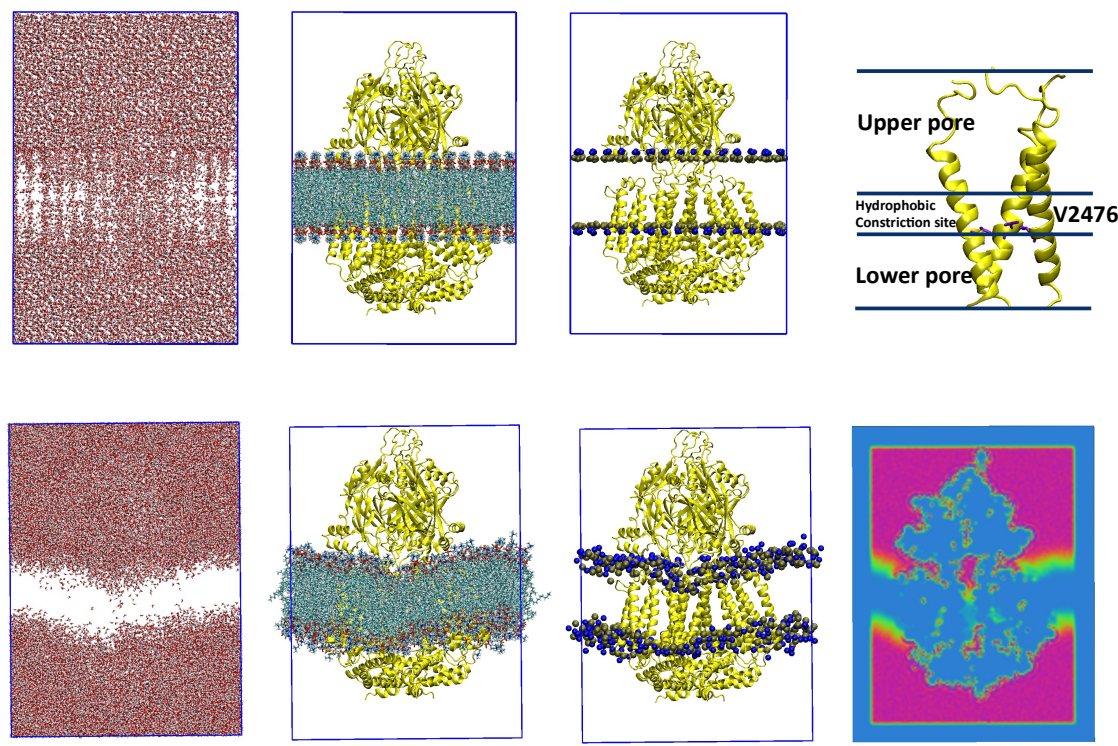
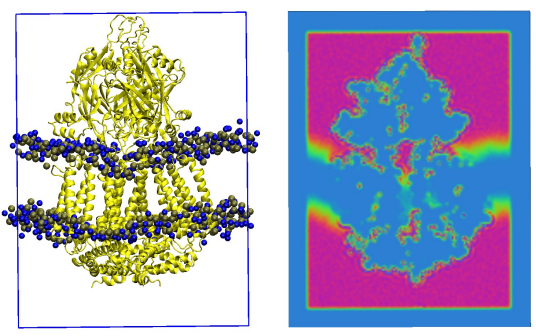

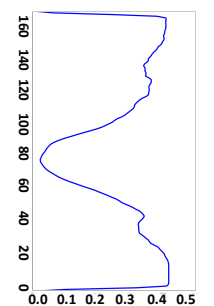

Density(1/周)

Figure 4. AA simulation of Piezo1 pore domain. a) AA initial system setup by components, and a close look of pore region divided into upper pore region, hydrophobic constriction site and lower pore region. $\mathbf{b}$ ) following the standard CHARMM-GUI protocol (using only headgroup restraint) during the equilibrium stage and fully release the restraint but protein backbone for the $1 \mu$ s production stage. Color scheme: oxygen atom in red, hydrogen atom in white, protein in yellow, V2476 residue in violet, phosphorus in brown, nitrogen in blue, hydrophobic tails in cyan. Water and lipids are shown in VMD licorice mode, the third column showing phosphorus and nitrogen atoms in VDW mode, protein is shown in new cartoon mode. The water density map is computed from the last 500ns for AA system and visualized with slice offset $=0.46$ along $y$-axis in VMD. The water density along $z$-axis is also plotted side by side. 


\section{Comparing AA model with the CG Rev-mapped AA model.}

The analysis above indicate that the CG whole lipid restraint equilibrium protocol yields similar pore hydration pattern as the system prepared directly from AA model. However, CG lipid headgroup-only restraint yields lipids in the pore rather than water in the pore. We hence ask whether the CG model with lipids in the pore, once reverse-mapped to the same AA force field, would converge to the similar hydration pattern as in the AA model? Two replicas of CG headgroup-only restraint system were reversemapped back to AA systems, called "CG Rev-mapped AA model", and continued AA production run for $200 \mathrm{~ns}$ to compare the number of water and lipid headgroups in the upper pore region with the original AA model described above (Figure 5). Note that one water molecule bead in CG model represents four TIP3 water molecules in AA model. Thus, there is a factor scale of 4 when comparing the number of water molecules found at the upper pore region in AA model with CG model. The original AA model pore contains 105 water molecules during the equilibrium stage, and later decreased to 71 water molecules during the production stage (the only difference between equilibrium and production stages is the removal of lipid restraint), while the CG Rev-mapped AA model initially only contains 20 water molecules during the equilibrium stage and later increased to 46 water molecules during the production stage, with the 3 lipids staying at the upper pore region for 200ns in the production run. In contrast, in both replicas of the original AA model $1 \mu$ s simulations, there was no lipid headgroup occluding the pore. Thus, the simulations conducted in this study reproduced unambiguously the discrepancy from previous all-atom simulations of Piezo1: the model solvated directly from AA model has no lipid trapped in the pore during $8 \mu$ s production run(7), while reverse-mapped AA models all have lipids remained in the pore(8,9). 

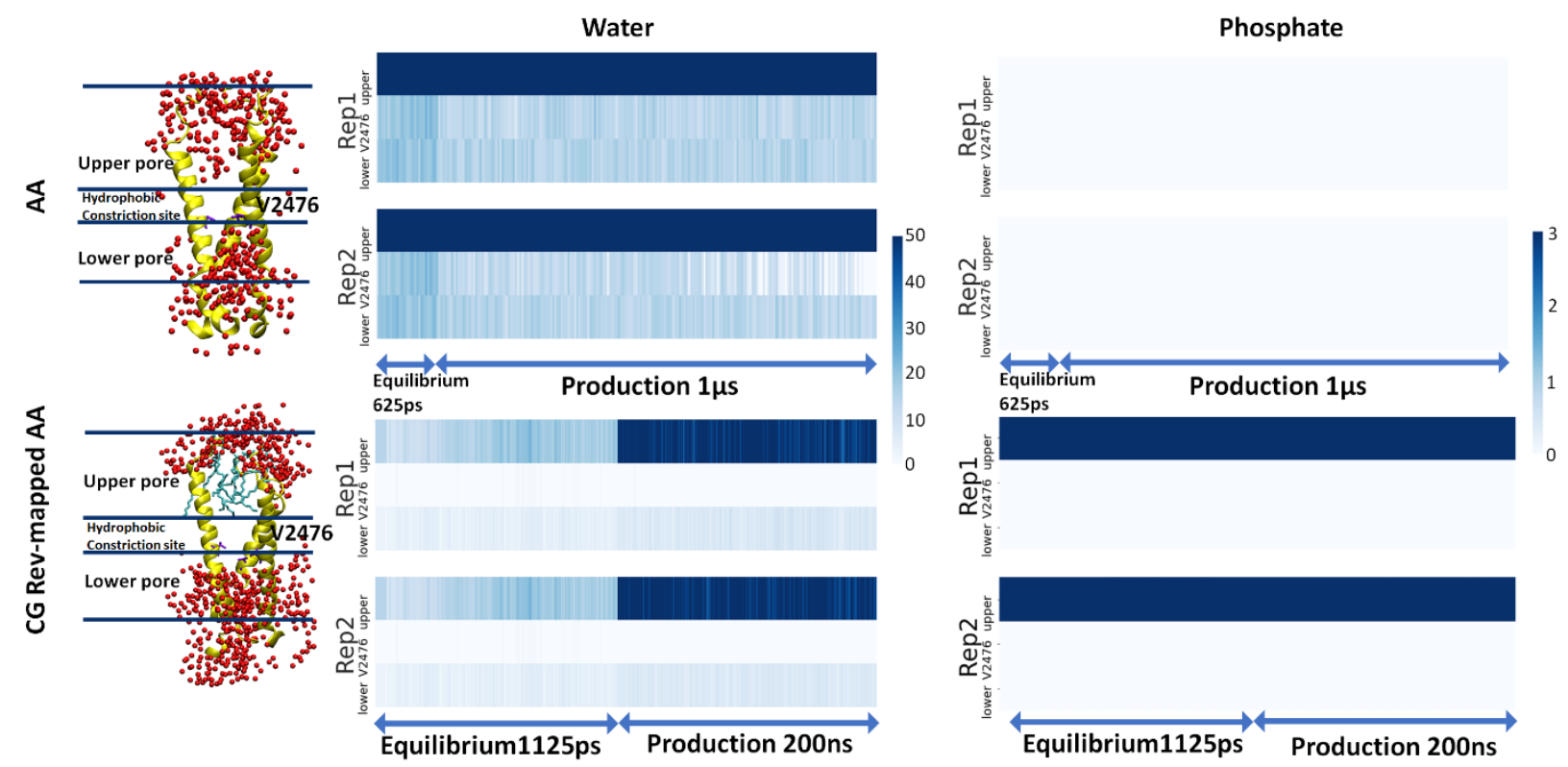

Figure 5. Number of water, lipid headgroup and tail in AA model and CG Rev-mapped AA model. Both two AA systems used the standard CHARMM-GUI equilibrium protocol with only lipid headgroup restraint for the bilayer during equilibrium stage, and fully released bilayer during the production stage. The production stage ran for $1 \mu \mathrm{s}$ for AA model and 200ns for CG Rev-mapped AA model with $10000 \mathrm{~kJ} / \mathrm{mol} / \mathrm{nm}^{2}$ position restraints on protein backbone. Each restraint system ran for two replicas with the same protocol. Color scheme: oxygen atom in red, V2476 residue in violet, protein in yellow, lipids in cyan, all other components are not shown here.

\section{Absolute Binding Free Energy of Lipids in Piezo1 Pore}

The results from CG and AA simulations indicate that the initial solvation algorithm and the type of the lipid restraint during equilibrium can result a drastically different hydration pattern in the Piezo1 pore and led to the presence of lipids in the pore. This difference introduced during equilibrium persisted throughout our multiple microseconds of AA simulations. Thus, we hypothesize that the lipids are kinetically trapped in the pore. If the free energy barrier for lipid exiting the pore is too high, spontaneous solvation of the pore will not occur in the timescale of AA simulations. To test this hypothesis, we seek to compute the absolute binding free energy of pore lipids. Here, absolute free energy is defined as the free energy difference between lipids in the pore and lipids in a homogenous POPC bilayer.

FEP $/ \lambda$ replica-exchange molecular dynamics (FEP/ $\lambda$-REMD) was used to the binding free energy of pore lipids (Figure 6a). Absolute binding free energy calculations require decoupling the lipids in protein binding site and in bulk bilayer (double decoupling scheme), as shown in thermodynamic circle is drawn 
in Figure 6b. For the three POPC in the pore, $128 \lambda$ intermediates were used, and the total number of perturbed atoms is 402. Because the phospholipids in the pore have high flexibility and mobility, we adopt the flat-bottom types of restraints previously used for GPCR-cholesterol binding calculation(17). Briefly, the ensemble distribution of protein-lipid distance and lipid conformational root-mean-squared-deviation (RMSD) from unbiased simulations are used to setup the upper-boundary of the flat-bottom harmonic restraints. This is to ensure unbiased sampling of pore lipids when the lipids were fully coupled $(\lambda=1)$. In addition, other lipid molecules near the pore were prevented from entering the pore during the perturbation, thus ensuring the sampling of the true unbound state in which pore is hydrated when $\lambda=$ 0 (Figure S6). Likewise, the flat-bottom harmonic restraints used for decoupling a POPC lipid in bilayer are also defined by the configurational space sampled by a random lipid in its leaflet (leaflet flip-flop is unlikely for POPC). The detailed methods and convergence analysis are provided in Methods section. The total absolute binding free energy of three lipids in the pore is $+45.9 \pm 0.9 \mathrm{kcal} / \mathrm{mol}$, which supports our hypothesis that lipids in the nonconducting Piezo1 pore were introduced during initial equilibrium step and kinetically trapped in this thermodynamically unfavorable state within microseconds.

One important requirement for accurate binding free energy prediction is that the first and last states of FEP should capture the same conformational ensemble of the true end states (apo and holo) from unbiased simulations. To double check this, we compared the water and lipid distribution patterns from unbiased simulations of $A A$ model and $C G$ rev-mapped $A A$ model with the results from the FEP trajectories of $\lambda=0$ and $\lambda=1$. Figure $\mathbf{S} 6$ shows the higher number of water molecules and absence of lipid headgroup in the pore at fully uncoupled state $(\lambda=0)$ agree well with the numbers from the AA equilibrated simulation. Likewise, the lower number of water and three lipid headgroups in the pore are also consistent between fully couple FEP state $(\lambda=1)$ and CG reverse-mapped AA simulation with headgroup-only restraint during equilibrium.

From the structure point of view, the upper pore region solved in cryo-EM structure is wide (radius 4 7 $\AA$ (Figure 1) and consists of only hydrophobic residues (residues shown in pink licorice model, Figure 2). The closest charged residue near lipid headgroups is more than $5 \AA$ away (K2329 at the bottom of cap domain). Thus, it is perhaps not surprising those headgroups do not bind to the pore favorably. However, while lipid tails are seen to fluctuate in and out of the upper and lower pore region frequently, lipid headgroups are trapped in the pore throughout the simulations. Although the exact free energy barrier for lipid exiting the pore is unknown, it is unlikely to occur during microseconds of AA simulations, thus could compromise the interpretation of MD simulation results. 
a)

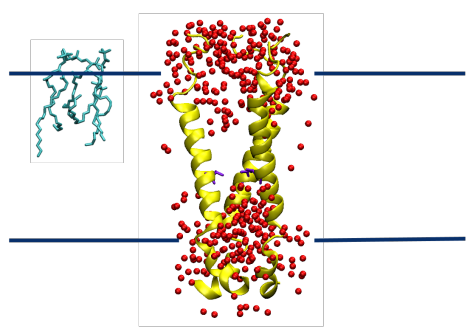

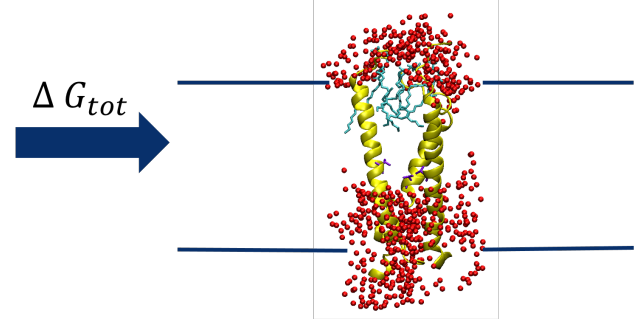

b)

$$
\Delta G_{t o t}=\Delta G_{F E P}^{s i t e}+\Delta G_{R M S D}^{g a s}+\Delta G_{g e o m}^{g a s}-\Delta G_{F E P}^{\text {bilayer }}
$$
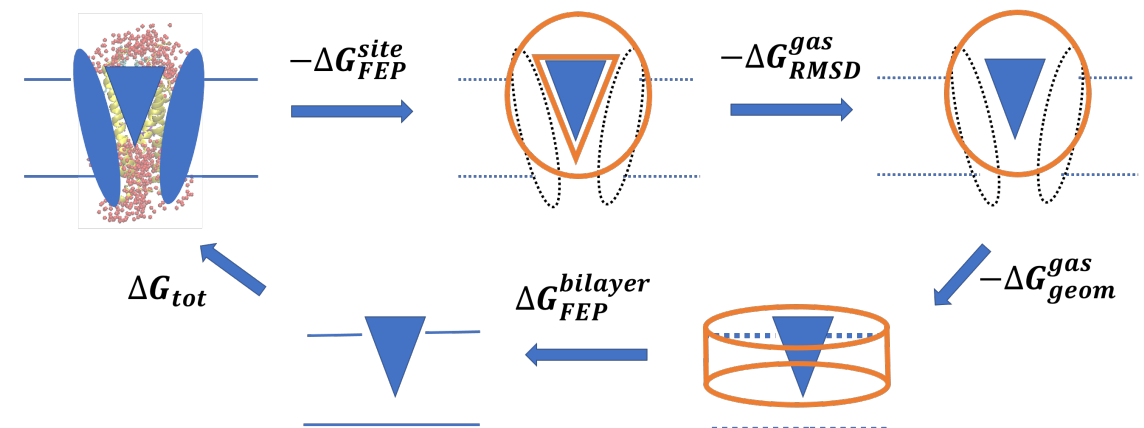

Flat-bottom harmonic restraints on:

lipid RMSD

binding site volume

monolayer volume

Figure 6. Binding free energy calculation of lipids in Piezo1 pore. a) The absolute binding free energy of lipids in Piezo1 pore corresponds to the reversible work to move the lipids from the bilayer to the pore. Color scheme: water oxygen atom in red, pore helix in yellow, lipids in cyan, V2476 residue in violet and all other components are not shown here. b) Graphic presentation of the thermodynamic circle of FEP protocol (see methods).

\section{DISCUSSION}

Protein-lipid interactions have drawn unprecedented attention in recent years, thanks to the increasing number of high-resolution membrane protein structures solved in lipid environment(18). The identification of lipid-like electron densities in these structures, coupled with functional studies, constitute a powerful experimental approach to investigate the contribution of lipids to channel gating(19). Alternatively, CG simulations using Martini force field has been successful in capturing lipid-protein interaction dynamics $(20,21)$, and estimating lipid binding free energy and kinetics $(22,23)$, thanks to faster lipid diffusion and smaller numbers of degrees of freedom compared to AA models. For studying the conformational changes of large membrane proteins embedded in curved and/or heterogenous membrane, a CG-equilibrium-AA-production protocol provides an efficient way to accelerate the 
convergence of lipids distribution before investigating protein dynamics with an atomistic resolution. While this multiscale approach has been successfully applied to many MD simulation studies(24), it is important to keep in mind that the slower AA sampling could be trapped in a free energy minimum generated from CG sampling. Indeed, we found here that once the lipid headgroups enter the fenestrated upper pore region of the Piezo1 pore during CG runs, these lipids become kinetically trapped in subsequent $\mathrm{AA}$ simulations.

Discrepancy in pore lipid density have been reported by structural biologists when comparing among different protein purification and reconstitution approaches(25). Here, the discrepancy in pore lipid density from MD simulations is partially due to the topology of the pore. Piezo1 is a homotrimer. The three inner pore helices form an hourglass shape with a hydrophobic constriction site formed by three V2476, and three fenestrations above and below this constriction site(8) (Figure 1). In CG model preparation, the protein is first embedded in a bilayer and then water beads are added above and below the bilayer, but not in transmembrane protein crevices such as channel pores. In the equilibrium step, both the protein and lipid headgroups are restrained to allow water molecules to diffuse freely and to fill in empty space. In principle, this setup should allow full hydration of the pore if the pore walls are sealed against lipids. If not, adjacent lipids enter the pore before the pore gets fully hydrated. Hence, this artifact can be easily eliminated by restraining whole lipids during initial water diffusion. Indeed, this artifact is not seen in our tested AA model because the AA solvation algorithm adds water in the pore before the equilibrium step. By restraining whole lipids during CG equilibrium, we obtain similar results as those obtained using AA equilibrium, including our previous $8 \mu$ s-long simulation(7), that is the absence of whole lipids in the pore.

Binding free energy calculations constitutes a robust method to determine the stability of protein bound lipids. The challenge in using alchemical approaches for protein-lipid binding in AA models is the sheer size and flexibility of the long fatty acid aliphatic chains. Although free energy is independent of the alchemical perturbation path (if the contribution of the restraint is properly accounted for), the phase space confined by geometric and conformational restraints on bound lipids needs to be small enough to ensure convergence but also large enough to sample all relevant binding poses. Here, we show that a set of flat-bottom harmonic restraints(17) are advantageous in computing absolute binding free energies of highly flexibility phospholipids. This FEP protocol allows unbiased sampling of lipids in the binding site and in the bulk bilayer when they are fully coupled, and also uses less number of restraints than the Boresch- 
style restraints (i.e., six rigid-body restraints)(26) commonly applied for computing absolute binding free energies of small molecules.

This study aimed to probe the mechanism by which lipids enter the Piezo1 pore during MD simulations. Although our data suggests whole lipids are not thermodynamically stable in the pore, by no means this study excludes the contribution of lipids to Piezo1 function. For instance, although proteinlipid interactions are dynamic and likely depend on protein conformation, here the non-conducting Piezo1 conformation had to be restrained to rigorously compare multiple equilibrium protocols. In addition, only POPC lipids were investigated here to serve as a baseline for investigating more complex protein-lipid interactions in heterogenous bilayer. Indeed, many lipids are known to modulate Piezo1 function such as PIP2(27), PS(28), margaric acid-enriched phospholipids(29, 30), and sterols(31-33). Understanding the role of lipids to the function of mechanosensitive ion channels remain a central question in the field of mechanobiology. Eliminating possible computational artifacts is an important step toward this goal.

\section{METHODS}

\section{CG simulation setup and protocols}

Atomistic model of nonconducting piezo1 pore (residue V1976 to R2546) was truncated from the our previously Piezo1 model built from cryo-EM structure (PDB 6B3R) (8). The CG simulation was executed in GROMACS (version 2016.4) simulation package with the standard Martini v2.2 force field parameter settings $(2,34)$. The CG models were built up with membrane using INSANE (INSert membrane) CG building tool(13). All lipid models and parameters used in this study followed the MARTINI v2.0 lipids(35). The overall workflow of the simulations included the initial construction of the Piezo1 embedded membrane, energy minimization, isothermal- isochoric (NVT) and isothermal-isobaric (NPT) equilibration runs, and NPT production runs. In detail, each system followed the standard CHARMM-GUI equilibrium protocol(36, 37). A cut-off of $1.2 \mathrm{~nm}$ was used for calculating both the electrostatic and van der Waals interaction terms; the potential-shift-Verlet algorithm was applied to take care of both interactions by smoothly shifting beyond the cutoff. Coulomb interactions were calculated using the reaction-field algorithm implemented in GROMACS. The neighbor list was updated every 20 steps using a neighbor list cutoff equal to $1.2 \mathrm{~nm}$ for short-range van der Waals. The temperature for each group (protein, membrane, ion, and water) was kept constant at $310.15 \mathrm{~K}$ using the velocity rescale coupling algorithm with $1 \mathrm{ps}$ time constant. In the NPT equilibration step, semi-isotropic pressure coupling was applied using the Parrinello-Rahman algorithm, with a pressure of 1 bar independently in the cross-section of the membrane and perpendicular to the membrane with the compressibility of $3.0 \times 10^{-4}$ bar $^{-1}$. The relaxation time constant for pressure coupling was set to 10.0 ps. Three-dimensional periodic boundary conditions were used. The CG system was first minimized using 5000 steepest descent cycles. Following minimization, in the first equilibration stage, a 1250 ps NVT solvent heating simulation ran to attain a target temperature of $310.15 \mathrm{~K}$ with a timestep of 10fs. A time step of $5 \mathrm{fs}$ was used for the following NPT simulations due to high positional restraint on 
protein backbone. Positional restraints were applied to backbone of protein residues with a force constant of $10000 \mathrm{~kJ} / \mathrm{mol} / \mathrm{nm}^{2}$ over all the stages too. In CG model, we tested two kinds of additional lipid restraints: one is only restraining positional restraints on phosphate headgroup beads of membrane lipid POPC residues, and another is restraining positional restraints on whole POPC beads (details in Table S1a). The first and second stage equilibrations were each run for a duration of 1250 ps each with lipid positional restraints of $5000 \mathrm{~kJ} / \mathrm{mol} / \mathrm{nm}^{2}$. Starting with the third equilibration stage, the duration of simulations was still 1250ps and an NPT ensemble was employed For the third and fourth stage, force constants for lipid POPC beads were reduced to $3000 \mathrm{~kJ} / \mathrm{mol} / \mathrm{nm}^{2}$. In the fifth stage, positional restraint force constants for lipid POPC were reduced to $1000 \mathrm{~kJ} / \mathrm{mol} / \mathrm{nm}^{2}$. In the sixth equilibration stage, membrane positional restraints were removed entirely, where the system was relaxed in a 5 ns equilibrium step using the Parrinello-Rahman barostat with a relaxation time constant equal to $10.0 \mathrm{ps}$. Two replicas were also conducted the same production stage with the same coordinates but different velocities for $1 \mu \mathrm{s}$.

\section{AA simulation setup and protocols}

In AA model, GROMACS commend "gmx solvate" was used to add water molecules. Water molecules were deleted from the box if the distance between any atom of the solute molecule(s) and any atom of the solvent molecule is less than the sum of the scaled van der Waals radii of both atoms which is smaller than a standard water bead of $5 \AA(12,16)$. To compare with CG MD simulations, this AA system was first minimized using 5000 steepest descent cycles in GROMACS 2016.4 package(34), and then underwent six stages of thermal equilibrium phase at $310.15 \mathrm{~K}$. All the stages and in both AA model and reverse-mapped AA model, a non-bonded cut-off $1.2 \mathrm{~nm}$ was employed for both the electrostatic and van der Waals interaction terms. The potential-shift-Verlet algorithm was applied to take care of both interactions by smoothly shifting beyond the cutoff. Coulomb interactions were calculated using PME algorithm implemented in GROMACS. Positional restraints were applied to backbone of protein residues with a force constant of $10000 \mathrm{~kJ} / \mathrm{mol} / \mathrm{nm}^{2}$ over all the stages (details in Table S1b). For all simulation stages, the neighbor list was updated every 20 steps using a neighbor list cutoff equal to $1.2 \mathrm{~nm}$ for short-range van der Waals and temperature control was accomplished using velocity rescale coupling algorithm with 1 ps time constant. The LINCS algorithm was used to constrain bonds involving hydrogen(38). In the NPT equilibration step, pressure regulation was accomplished by utilization of a semi-isotropic ParrinelloRahman barostat with a pressure of 1 bar independently in the cross-section of the membrane and perpendicular to the membrane with the compressibility of $4.5 \times 10^{-5} \mathrm{bar}^{-1}$. The relaxation time constant for pressure coupling was set to $5.0 \mathrm{ps}$. Following minimization, in the first equilibration stage, a $125 \mathrm{ps}$ NVT solvent heating simulation ran to attain a target temperature of $310.15 \mathrm{~K}$. Phosphate atoms of membrane POPC residues were restrained with a force constant of $5000 \mathrm{~kJ} / \mathrm{mol} / \mathrm{nm}^{2}$. Dihedral restraints were also employed to ensure retention of backbone phi-psi angles during minimization with 2000 $\mathrm{kJ} / \mathrm{mol} / \mathrm{radian}^{2}$ force constant. The second equilibration stage followed an identical setup of $125 \mathrm{ps}$ with the sole exception that the force constant of the dihedral phi-psi restraint was also reduced to 1000 $\mathrm{kJ} / \mathrm{mol} / \mathrm{radian}^{2}$. Starting with the third equilibration stage, an NPT ensemble was employed. For the third stage of $125 \mathrm{ps}$, force constants for phosphate atoms positional restraints were reduced to 3000 $\mathrm{kJ} / \mathrm{mol} / \mathrm{nm}^{2}$. The dihedral phi-psi restraints were also dropped to $500 \mathrm{~kJ} / \mathrm{mol} / \mathrm{radian}^{2}$. In the fourth stage, 
positional restraint force constants for phosphate atoms were maintained at $3000 \mathrm{~kJ} / \mathrm{mol} / \mathrm{nm}^{2}$ and phipsi backbone dihedral restraint force constants were reduced to $200 \mathrm{~kJ} / \mathrm{mol} / \mathrm{radian}^{2}$. From the fourth to sixth stages, AA model ran for $125 \mathrm{ps}$ each and reverse-mapped AA model for 250ps, respectively. In the fifth stage, positional restraint force constants for phosphate atoms were reduced to $1000 \mathrm{~kJ} / \mathrm{mol} / \mathrm{nm} 2$ and phi-psi backbone dihedral restraint force constants were reduced to $100 \mathrm{~kJ} / \mathrm{mol} / \mathrm{radian}^{2}$. In the sixth equilibration stage, membrane positional restraints and phi-psi backbone dihedral restraints were removed entirely. Simulation timestep was set as $1.0 \mathrm{fs}$ due to high positional restraint on protein backbone. Later, for $A A$ model, a $1 \mu$ s equilibration production run with only positional restraint on backbone atoms of $100 \mathrm{kcal} / \mathrm{mol} / \AA^{2}$ was conducted using PEMED CUDA module of Amber18 packages(39) for two replicas with the same coordinates but different velocities (details in Table S1c). Other parameters are the same with GROMACS setting except temperature control was done using Langevin thermostat with a gamma parameter (friction coefficient) of $1.0 \mathrm{ps}^{-1}$, with pressure coupling using a semi-isotropic Monte-Carlo barostat with a target pressure of the 1.0 bar and constant zero membrane surface tension and the SHAKE algorithm(40) was used to constrain bonds involving hydrogen. The trajectories were saved every $1 \mathrm{~ns}$ for $1 \mu$ s-production run. For reverse-mapped AA model, 200ns production run of two reps were performed in Amber18 packages with only positional restraint on backbone atoms of 100 $\mathrm{kcal} / \mathrm{mol} / \AA^{2}$. Trajectories of 200ns were saved every 200ps.

\section{Free Energy Perturbation Protocol for Lipid-Protein Binding}

The absolute binding free energy of lipids in Piezo1 pore corresponds to the thermodynamic reversible work to move the lipids from the bilayer to the binding site, which is the upper pore region in this case. The dissociate constant is $K_{d}=\frac{[P][L]}{[P L]}$, where $[P],[L],[P L]$ are the equilibrium concentrations of lipid, protein, and bound complex, respectively. Since we are only concerning the final bound state, it is convenient to treat the cluster of three POPC lipids in the pore as a single ligand regardless of the order of their entering the pore. Unlike small-molecule ligands, once bound to their protein target, display a well-defined binding mode, lipids bound to protein pore or protein surface can display larger mobility and conformational flexibility. To ensure the FEP/ $\lambda$-REMD sampled the relevant lipid conformations ensemble in the bound state, flat-bottom harmonic potentials(17) were introduced to the bound distance and bound conformational ensemble of the lipids relative to the binding site when the lipids are gradually (128 $\lambda$ intermediates) decoupled from the binding site. Unbiased trajectories were used to compute the ensemble distribution of (1) the distance R between center of mass (COM) of three lipids and the pore, and (2) lipid conformational RMSD after rigid-body alignment of the pore at frame 0 . The upper-limit of the ensemble distribution of R and RMSD from unbiased simulations were used to setup the upperboundary of the flat-bottom harmonic restraint for the lipid RMSD and for distance (force constant of 100 $\left.\mathrm{kcal} / \mathrm{mol} / \AA^{2}\right)$ so that when the lipids were fully coupled $(\lambda=1)$, their free energy contributions of the $R$ and RMSD restraints were negligible. In addition, to ensure that FEP/ $\lambda$-REMD sampled the two end states properly, lipids outside the pore were prevented from entering the pore during FEP so that water can enter the pore during the decoupling stage. This was achieved by a RMSD restraint on the center-of-mass of a lipid (selected atom names are N C2 C218 C316) between current frame and initial frame to prevent binding without otherwise biasing the statistical distribution of unbound lipid molecules. An example of 
NAMD Colvars restraint file for all the required restraint setting is provided at https://github.com/reneejiang/pore-lipids.

According to Figure $6 \mathrm{~b}$, the absolute binding free energy can be obtained using $\Delta G_{\text {tot }}=\Delta G_{F E P}^{\text {site }}$ $+\Delta G_{R M S D}^{\text {gas }}+\Delta G_{\text {geom }}^{\text {gas }}-\Delta G_{F E P}^{\text {bilayer }}$, where $\Delta G_{F E P}^{\text {site }}$ denotes the contribution of decoupling bound lipids $(\lambda=1 \rightarrow 0)$. A total of $128 \lambda$ intermediates was used to ensure $>40 \%$ acceptance ratio between neighboring states. $\Delta G_{R M S D}^{g a s}$ denotes the contribution of flat-bottom harmonic RMSD restraint on conformational ensemble of lipids when lipids are fully uncoupled (i.e. in gas phase, $\lambda=0$ ). $\Delta G_{R M S D}^{\text {gas }}$ was calculated using NAMD Colvars module(41) with $20 \lambda$ values from 1.0 to 0.0 ( $\lambda=$ $1,0.9999,0.999,0.99,0.9,0.8,0.7,0.6,0.5,0.4,0.35,0.325,0.3,0.25,0.225,0.2,0.175,0.15,0.125,0)$. Each $\lambda$ was run for 200ps and errors were calculated from three replicas. $\Delta G_{\text {geom }}^{\text {gas }}$ denotes the entropic contribution, thus the volume ratio of fully uncoupled lipids in the binding site $\left(\frac{3}{4} \pi R^{3}\right)$ versus the sampled volume of uncoupled POPC in the bulk bilayer. $R$ is the upper boundary of flat-bottom distance restraint between lipids and Piezo1 pore. In a bulk bilayer with surface area $A$, a flat-bottom restraint was introduced on the $z$-axis distance of the POPC lipid headgroup COM from the upper leaflet midplane $Z_{R}$ so that the uncoupled POPC remains in the upper leaflet. In addition, an orientational restraint between the principal vector of the decoupled POPC lipid and the normal axis of bilayer $\theta$ is applied so that the uncoupled lipid remains similar orientation as in fully coupled state. Thus, the volume sampled by uncoupled lipid in bulk bilayer is $2 Z_{R} A(1-\operatorname{Cos} \theta) . \Delta G_{F E P}^{\text {bilayer }}$ is the free energy contribution of decoupling three individual POPC lipid from a POPC bilayer, which is computed as three folds of the free energy of decoupling a single lipid in the bilayer. Similar as in the binding site, the flat-bottom harmonic restraints on $Z_{R}$ and $\theta$ have zero contribution when the lipid is fully coupled. Two different POPC bilayer sizes, 40 per leaflet and 60 per leaflet, were tested and yield the $\Delta G_{F E P}^{\text {bilayer }}$ values within uncertainty (Figure S4).

To compute $\Delta G_{F E P}^{\text {site }}$ and $\Delta G_{F E P}^{\text {bilayer }}, \mathrm{FEP} / \lambda$-REMD implemented in NAMD Git-2021-06-02 program using generalized scalable multiple copy algorithms was used $(42,43)$. A total number of 128 replicas were used for the binding site and 64 replicas were used for bulk bilayers of size 40 and 60 lipids per leaflet. The "softcore" potentials were used to avoid end-point catastrophe $(44,45)$. Each replica in the FEP/ $\lambda$ REMD simulation represents a state along the coupling parameter, and periodic swap is attempted between neighboring replicas every 100 steps $(0.2 \mathrm{ps})$. The accuracy of FEP/ $\lambda$-REMD depends on the overlaps between two potential energy distributions, which can be measured by the acceptance ratio between replicas. Convergence was monitored by the time dependence of each predicted free energy term. This sampling time dependence provides an asymptotically unbiased estimator for each $\Delta G$. We considered the FEP/ $\lambda$-REMD simulation is converged when that last two 0.5 ns running average of free energy value fluctuates within $0.5 \mathrm{kcal} / \mathrm{mol}$ (Figure S2 and S4). The uncorrelated samples found in each replica are calculated using pymbar alchemical analysis toolbox (Figure S3)(46, 47).

The free energy contribution of each restraint is listed in Table S2. The acceptance ratio between each adject pair of 128 replicas is between $40 \%$ to $80 \%$ (Figure S1) and the number of uncorrelated samplings in each replica ranges from 40 to 1000 (Figure S3). The acceptance ratio between each adject pair of 64 replicas in the 60/leaflet POPC bilayer is $30 \%$ to $70 \%$ (Figure S5). The convergence of each FEP 
stage is shown in timeseries in Figure $\mathbf{S 2}$ and S4. The standard deviation of $\Delta G_{R M S D}^{g a s}$ was calculated from three replicas of simulations. The uncertainties of $\Delta G_{F E P}^{\text {site }}$ and $\Delta G_{F E P}^{\text {bilayer }}$ were computed from simple overlapping sampling method(47-49) implemented in pymbar(46, 47).

\section{Analysis of atoms/beads at Piezo1 pore regions}

The counting method for classifying atoms/beads inside pore regions was based on the MATLAB function "inpolyhedron"(50) which can efficiently classify whether a point inside of a 3D triangulated (faces/vertices) surface. With adding additional surfaces to build up a closed volume for upper pore region, hydrophobic constriction site and lower pore region along the z-axis, the time series of the number of atoms or beads inside each region were classified correctly and plotted. An example plot of the classification method is shown in Figure S7. The in-house MATLAB codes are provided at https://github.com/reneejiang/pore-lipids.

\section{CODE AVAILABILITY}

Input files for MD simulations and free energy calculations, as well as python2 scripts are publicly available at https://github.com/reneejiang/pore-lipids.

\section{ACKNOWLEDGMENTS}

This work was supported by NIH Grants GM130834 (Y.L. and J.L.). Computational resources were provided via the Extreme Science and Engineering Discovery Environment (XSEDE) allocation TG-MCB160119, which is supported by NSF grant number ACl-154862. Anton2 computer time was provided by the Pittsburgh Supercomputing Center (PSC) through NIH Grant R01-GM116961. The Anton2 machine at PSC was generously made available by D.E.Shaw Research.

\section{AUTHOR CONTRIBUTIONS}

W.J. performed all MD simulations and data analysis. Y.L designed and supervised the project. W.J., and Y.L and J.L wrote the paper together.

\section{REFERENCE}


1. de Jong, D. H., G. Singh, W. D. Bennett, C. Arnarez, T. A. Wassenaar, L. V. Schafer, X. Periole, D. P. Tieleman, and S. J. Marrink. 2013. Improved parameters for the martini coarse-grained protein force field. Journal of chemical theory and computation 9(1):687-697.

2. Marrink, S. J., H. J. Risselada, S. Yefimov, D. P. Tieleman, and A. H. De Vries. 2007. The MARTINI force field: coarse grained model for biomolecular simulations. The journal of physical chemistry B 111(27):7812-7824.

3. Wassenaar, T. A., K. Pluhackova, R. A. Böckmann, S. J. Marrink, and D. P. Tieleman. 2014. Going backward: a flexible geometric approach to reverse transformation from coarse grained to atomistic models. Journal of chemical theory and computation 10(2):676-690.

4. Ge, J., W. Li, Q. Zhao, N. Li, M. Chen, P. Zhi, R. Li, N. Gao, B. Xiao, and M. Yang. 2015. Architecture of the mammalian mechanosensitive Piezo1 channel. Nature 527(7576):64-69.

5. Coste, B., J. Mathur, M. Schmidt, T. J. Earley, S. Ranade, M. J. Petrus, A. E. Dubin, and A. Patapoutian. 2010. Piezo1 and Piezo2 are essential components of distinct mechanically activated cation channels. Science 330(6000):55-60.

6. Guo, Y. R., and R. MacKinnon. 2017. Structure-based membrane dome mechanism for Piezo mechanosensitivity. Elife 6:e33660.

7. Botello-Smith, W. M., W. Jiang, H. Zhang, A. D. Ozkan, Y.-C. Lin, C. N. Pham, J. J. Lacroix, and Y. Luo. 2019. A mechanism for the activation of the mechanosensitive Piezo1 channel by the small molecule Yoda1. Nature Communications 10(1):4503.

8. Jiang, W., J. S. Del Rosario, W. Botello-Smith, S. Zhao, Y.-c. Lin, H. Zhang, J. Lacroix, T. Rohacs, and Y. L. Luo. 2021. Crowding-induced opening of the mechanosensitive Piezo1 channel in silico. Communications Biology 4(1):84.

9. De Vecchis, D., D. J. Beech, and A. C. Kalli. 2021. Molecular dynamics simulations of Piezo1 channel opening by increases in membrane tension. Biophysical Journal 120(8):1510-1521.

10. Reddy, B., N. Bavi, A. Lu, Y. Park, and E. Perozo. 2019. Molecular basis of force-from-lipids gating in the mechanosensitive channel MscS. Elife 8:e50486.

11. Brohawn, S. G., E. B. Campbell, and R. MacKinnon. 2014. Physical mechanism for gating and mechanosensitivity of the human TRAAK K+ channel. Nature 516(7529):126-130.

12. Berendsen, H. J., D. van der Spoel, and R. van Drunen. 1995. GROMACS: a message-passing parallel molecular dynamics implementation. Computer physics communications 91(1-3):43-56.

13. Wassenaar, T. A., H. I. Ingólfsson, R. A. Böckmann, D. P. Tieleman, and S. J. Marrink. 2015. Computational lipidomics with insane: a versatile tool for generating custom membranes for molecular simulations. Journal of chemical theory and computation 11(5):2144-2155.

14. Smart, O. S., J. M. Goodfellow, and B. A. Wallace. 1993. The pore dimensions of gramicidin A. Biophys J 65(6):2455-2460.

15. Humphrey, W., A. Dalke, and K. Schulten. 1996. VMD: visual molecular dynamics. J Mol Graph 14(1):33-38, 27-38.

16. Hess, B., D. van der Spoel, and E. Lindahl. Gromacs Manual. Energy 2:1.3.

17. Salari, R., T. Joseph, R. Lohia, J. Hénin, and G. Brannigan. 2018. A streamlined, general approach for computing ligand binding free energies and its application to GPCR-bound cholesterol. Journal of chemical theory and computation 14(12):6560-6573.

18. Mio, K., and C. Sato. 2018. Lipid environment of membrane proteins in cryo-EM based structural analysis. Biophys Rev 10(2):307-316.

19. Thompson, M. J., and J. E. Baenziger. 2020. Ion channels as lipid sensors: from structures to mechanisms. Nature Chemical Biology 16(12):1331-1342.

20. Corradi, V., E. Mendez-Villuendas, H. I. Ingólfsson, R.-X. Gu, I. Siuda, M. N. Melo, A. Moussatova, L. J. DeGagné, B. I. Sejdiu, and G. Singh. 2018. Lipid-protein interactions are unique fingerprints for membrane proteins. ACS central science 4(6):709-717. 
21. Buyan, A., C. D. Cox, J. Barnoud, J. Li, H. S. M. Chan, B. Martinac, S. J. Marrink, and B. Corry. 2020. Piezo1 Forms Specific, Functionally Important Interactions with Phosphoinositides and Cholesterol. Biophys J 119(8):1683-1697.

22. Hedger, G., D. Shorthouse, H. Kolds $\varnothing$, and M. S. Sansom. 2016. Free energy landscape of lipid interactions with regulatory binding sites on the transmembrane domain of the EGF receptor. The Journal of Physical Chemistry B 120(33):8154-8163.

23. Corey, R. A., O. N. Vickery, M. S. Sansom, and P. J. Stansfeld. 2019. Insights into membrane protein-lipid interactions from free energy calculations. Journal of chemical theory and computation 15(10):5727-5736.

24. Pluhackova, K., T. A. Wassenaar, and R. A. Bockmann. 2013. Molecular dynamics simulations of membrane proteins. Methods Mol Biol 1033:85-101.

25. Guo, Y. 2021. Detergent-free systems for structural studies of membrane proteins. Biochem Soc Trans 49(3):1361-1374.

26. Boresch, S., F. Tettinger, M. Leitgeb, and M. Karplus. 2003. Absolute binding free energies: A quantitative approach for their calculation. J Phys Chem B 107(35):9535-9551.

27. Borbiro, I., D. Badheka, and T. Rohacs. 2015. Activation of TRPV1 channels inhibits mechanosensitive Piezo channel activity by depleting membrane phosphoinositides. Sci Signal 8(363):ra15.

28. Tsuchiya, M., Y. Hara, M. Okuda, K. Itoh, R. Nishioka, A. Shiomi, K. Nagao, M. Mori, Y. Mori, J. Ikenouchi, R. Suzuki, M. Tanaka, T. Ohwada, J. Aoki, M. Kanagawa, T. Toda, Y. Nagata, R. Matsuda, Y. Takayama, M. Tominaga, and M. Umeda. 2018. Cell surface flip-flop of phosphatidylserine is critical for PIEZO1-mediated myotube formation. Nat Commun 9(1):2049.

29. Romero, L. O., A. E. Massey, A. D. Mata-Daboin, F. J. Sierra-Valdez, S. C. Chauhan, J. F. CorderoMorales, and V. Vasquez. 2019. Dietary fatty acids fine-tune Piezo1 mechanical response. Nat Commun 10(1):1200.

30. Romero, L. O., R. Caires, A. R. Nickolls, A. T. Chesler, J. F. Cordero-Morales, and V. Vasquez. 2020. A dietary fatty acid counteracts neuronal mechanical sensitization. Nat Commun 11(1):2997.

31. Poole, K., R. Herget, L. Lapatsina, H. D. Ngo, and G. R. Lewin. 2014. Tuning Piezo ion channels to detect molecular-scale movements relevant for fine touch. Nat Commun 5:3520.

32. Qi, Y., L. Andolfi, F. Frattini, F. Mayer, M. Lazzarino, and J. Hu. 2015. Membrane stiffening by STOML3 facilitates mechanosensation in sensory neurons. Nat Commun 6:8512.

33. Ridone, P., E. Pandzic, M. Vassalli, C. D. Cox, A. Macmillan, P. A. Gottlieb, and B. Martinac. 2020. Disruption of membrane cholesterol organization impairs the activity of PIEZO1 channel clusters. J Gen Physiol 152(8).

34. Pronk, S., S. Páll, R. Schulz, P. Larsson, P. Bjelkmar, R. Apostolov, M. R. Shirts, J. C. Smith, P. M. Kasson, and D. Van Der Spoel. 2013. GROMACS 4.5: a high-throughput and highly parallel open source molecular simulation toolkit. Bioinformatics 29(7):845-854.

35. López, C. A., Z. Sovova, F. J. van Eerden, A. H. de Vries, and S. J. Marrink. 2013. Martini force field parameters for glycolipids. Journal of chemical theory and computation 9(3):1694-1708.

36. Qi, Y., H. I. Ingólfsson, X. Cheng, J. Lee, S. J. Marrink, and W. Im. 2015. CHARMM-GUI martini maker for coarse-grained simulations with the martini force field. Journal of chemical theory and computation 11(9):4486-4494.

37. Jo, S., T. Kim, V. G. Iyer, and W. Im. 2008. CHARMM - GUI: a web - based graphical user interface for CHARMM. Journal of computational chemistry 29(11):1859-1865.

38. Hess, B., H. Bekker, H. J. Berendsen, and J. G. Fraaije. 1997. LINCS: a linear constraint solver for molecular simulations. Journal of computational chemistry 18(12):1463-1472.

39. Case, D., I. Ben-Shalom, S. Brozell, D. Cerutti, T. Cheatham III, V. Cruzeiro, T. Darden, R. Duke, D. Ghoreishi, and M. Gilson. AMBER 18; 2018. University of California, San Francisco. 
40. Ryckaert, J.-P., G. Ciccotti, and H. J. Berendsen. 1977. Numerical integration of the cartesian equations of motion of a system with constraints: molecular dynamics of n-alkanes. Journal of computational physics 23(3):327-341.

41. Fiorin, G., M. L. Klein, and J. Hénin. 2013. Using collective variables to drive molecular dynamics simulations. Molecular Physics 111(22-23):3345-3362.

42. Jiang, W., M. Hodoscek, and B. Roux. 2009. Computation of absolute hydration and binding free energy with free energy perturbation distributed replica-exchange molecular dynamics. Journal of chemical theory and computation 5(10):2583-2588.

43. Jiang, W., J. C. Phillips, L. Huang, M. Fajer, Y. Meng, J. C. Gumbart, Y. Luo, K. Schulten, and B. Roux. 2014. Generalized scalable multiple copy algorithms for molecular dynamics simulations in NAMD. Computer physics communications 185(3):908-916.

44. Beutler, T. C., A. E. Mark, R. C. Vanschaik, P. R. Gerber, and W. F. Vangunsteren. 1994. Avoiding Singularities and Numerical Instabilities in Free-Energy Calculations Based on Molecular Simulations. Chem Phys Lett 222(6):529-539.

45. Zacharias, M., T. P. Straatsma, and J. A. Mccammon. 1994. Separation-Shifted Scaling, a New Scaling Method for Lennard-Jones Interactions in Thermodynamic Integration. J Chem Phys 100(12):9025-9031.

46. Shirts, M. R., and J. D. Chodera. 2008. Statistically optimal analysis of samples from multiple equilibrium states. The Journal of chemical physics 129(12):124105.

47. Chodera, J. D., W. C. Swope, J. W. Pitera, C. Seok, and K. A. Dill. 2007. Use of the weighted histogram analysis method for the analysis of simulated and parallel tempering simulations. Journal of Chemical Theory and Computation 3(1):26-41.

48. Kong, A., P. McCullagh, X. L. Meng, D. Nicolae, and Z. Tan. 2003. A theory of statistical models for Monte Carlo integration. Journal of the Royal Statistical Society: Series B (Statistical Methodology) 65(3):585-604.

49. Lu, N., D. A. Kofke, and T. B. Woolf. 2004. Improving the efficiency and reliability of free energy perturbation calculations using overlap sampling methods. J Comput Chem 25(1):28-39.

50. Sven. 2021. Inpolyhedron- are points inside a triangulated volume? 